\title{
Delayed radiation-induced lumbosacral radiculoplexus neuropathy - a diagnostic dilemma
}

\author{
Rose M. Domingo-Horne*
}

VA Boston Healthcare System, Neurology Service/Division of Neuromuscular Medicine, Boston University School of Medicine, Instructor of Neurology Boston, Massachusetts, USA

\begin{abstract}
The delayed effects of neurotoxicity related to radiation therapy present a diagnostic challenge. It is essential for clinicians to recognize radiation-induced radiculoplexus neuropathy, which should also be discussed with patients as a potential iatrogenic complication of cancer treatment.
\end{abstract}

\section{Case}

A 70-year-old gentleman presented to our EMG laboratory in early 2015 for recurrent episodes of right lower extremity "buckling" and falls. His symptoms were gradual in onset and had been worsening over several weeks. His past medical history included ankylosing spondylitis, a decade-long history of poorly controlled diabetes mellitus (most recent $\mathrm{HbAlc}$ at that time $8.2 \%$ ), and remote prostate adenocarcinoma (T1c with perineural invasion, Gleason 3+4, PSA 4.36), for which he had received definitive radiotherapy in early 2008 (45 Gy whole pelvis, followed by serial coned-down boosts to the prostate and seminal vesicles; total dose 75.6 Gy). Radiotherapy was complicated by recurrent prostatitis, radiation-induced enteritis, and an associated partial small bowel obstruction in 2014.

He was found to have severe proximal right lower extremity weakness, with near-inability to flex at the hip or extend at the knee. There was moderate weakness in hip abduction and adduction noted, while knee flexion, ankle dorsiflexion, and ankle plantar flexion were preserved and unaffected. There was an area of hypesthesia along the right upper anterior thigh, as well as length-dependent bilateral lower extremity sensory loss and hyporeflexia. The distal lower extremities were edematous. The remainder of his neurological and general medical examinations at that time were unremarkable. An EMG was performed (Tables 1 and 2), with abnormalities taken within clinical context concerning for a severe proximal right lumbosacral radiculoplexopathy and underlying sensorimotor axon loss-predominant peripheral polyneuropathy.

He was admitted for additional workup and treatment. A pelvic MRI was notable for a T2/STIR hyperintense signal abnormality and enhancement involving the right pectineus, adductor magnus, iliacus, and psoas muscles, and faint enhancement of the right femoral and obturator nerves (Figure 1). For a presumed diagnosis of diabetic amyotrophy, he received 12 weeks of intravenous methylprednisolone and inpatient rehabilitation.

He was lost to follow-up until early 2016, when he presented with progressively worsening painless right leg weakness. He reported no subjective improvement either during or following his earlier treatment course with corticosteroids. His interim glycemic control had remained suboptimal (average HbAlc 8.5\%). He otherwise denied any significant change in his weight or general medical wellbeing. On reevaluation, he was found to have pronounced atrophy of the right quadriceps, continued proximal right leg weakness graded similarly to the year before, and the interim development of a nearcomplete right foot drop of uncertain chronicity. Sensory deficits were unchanged. The lower extremities were areflexic. A follow-up EMG again revealed abnormalities concerning for a severe right lumbosacral radiculoplexopathy, with interval worsening demonstrated by the extension of significant denervation changes to the distal right leg,

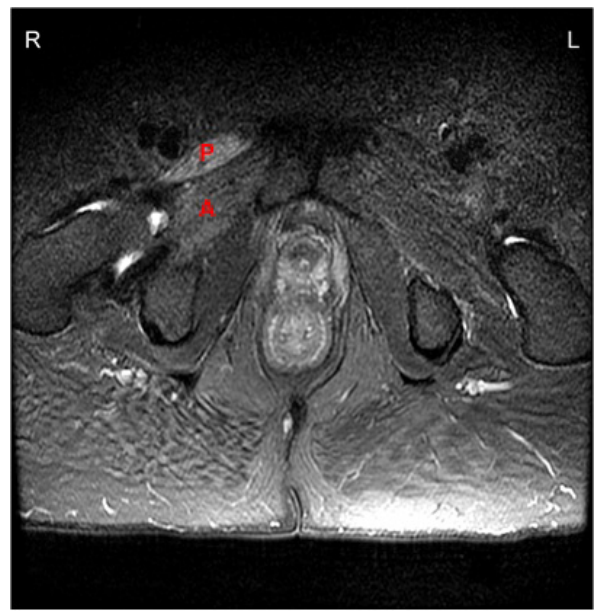

Figure 1. MRI of Pelvis (2015). Axial T2 hyperintense signal demonstrated, most prominent within the right pectineus $(\mathrm{P})$ and adductor magnus $(\mathrm{A})$ muscles.

Correspondence to: Rose M. Domingo-Horne, MD. VA Boston Healthcare System, Neurology Service / Division of Neuromuscular Medicine Boston University School of Medicine, Instructor of Neurology Boston, Massachusetts, USA, E-mail: Rose.Domingo-Horne@va.gov

Key words: cancer, lumbosacral, neuropathy, neurotoxicity, radiculoplexopathy, radiotherapy

Received: February 27, 2017; Accepted: March 20, 2017; Published: March 24, 2017 
and subclinical milder denervation changes extending to the distal left leg (Tables 3 and 4). He was readmitted, and a subsequent pelvic MRI revealed continuous denervation and fatty replacement of the bilateral pectineus, gluteus maximus and medius muscles, right obturator externus and internus muscles, and low lumbar paraspinal muscles. Radiation changes were seen within the sacrum. No definite nerve enhancement or space-occupying lesions were identified (Figure 2). Of note, MR imaging of the lumbar spine at each presentation revealed stable degenerative changes related to ankylosing spondylitis, most significant at the L4-L5 levels.

\section{Discussion}

Delayed radiation-induced lumbosacral radiculoplexus neuropathy is an under-recognized phenomenon, characterized by insidious and progressive lower motor neuron dysfunction in the absence of significant pain or sensory signs (which, if present, may be mild and develop late in the course of illness). Neurological deficits are typically asymmetric and unilateral, however bilateral involvement may occur. A gradual progression of deficits follows in a step-wise manner, owing to the pathophysiologic cycle of inflammation and sclerosis which underlies this condition [1].

Table 1. Nerve Conduction Studies - April 2015.

\begin{tabular}{|c|c|c|c|c|c|c|c|c|c|c|c|c|c|}
\hline \multirow[t]{2}{*}{ Nerve Stimulated } & \multirow[t]{2}{*}{ Stimulation Site } & \multirow[t]{2}{*}{ Recording Site } & \multicolumn{3}{|c|}{ Amplitude* } & \multicolumn{3}{|c|}{ Latency (ms) } & \multicolumn{3}{|c|}{ Conduction Velocity (m/s) } & \multirow[t]{2}{*}{ F wave Latency (ms) } & \multirow[t]{2}{*}{ Temperature $\left({ }^{\circ} \mathrm{C}\right)$} \\
\hline & & & RT & LT & NL & RT & LT & NL & RT & LT & NL & & \\
\hline \multirow[t]{3}{*}{ Peroneal (m) } & Ankle & EDB & 1.1 & & $\geq 2.0$ & 5.3 & & $\leq 6.6$ & & & & Not performed & \\
\hline & Below fibula & EDB & 0.6 & & & 13.9 & & & 37.2 & & $\geq 42.0$ & & \\
\hline & Lateral popliteal fossa & EDB & 0.6 & & & 16.6 & & & 36.9 & & $\geq 42.0$ & & 31.1 \\
\hline \multirow[t]{2}{*}{ Tibial (m) } & Ankle & AHB & 2.6 & & $\geq 2.0$ & 6.3 & & $\leq 6.6$ & & & & Not performed & \\
\hline & Popliteal fossa & AHB & 1.1 & & & 17.5 & & & 35.6 & & $\geq 42.0$ & & 31.1 \\
\hline Sural (s) & Calf & Posterior ankle & NR & & $\geq 5.0$ & NR & & $\leq 4.2$ & NR & & $\geq 42.0$ & & 31.1 \\
\hline
\end{tabular}

* Amplitude: motor in millivolts; sensory in microvolts

Key: m, motor study; s, sensory study; RT, right; LT, left; NL, normal; EDB, extensor digitorum brevis; AHB, abductor hallucis brevis; NR, no response

Note: sensory latencies are peak latencies

Table 2. Electromyography - April 2015

\begin{tabular}{|c|c|c|c|c|c|c|c|c|}
\hline \multirow[b]{2}{*}{ Muscle } & \multicolumn{3}{|c|}{ Spontaneous Activity } & \multicolumn{5}{|c|}{ Voluntary Motor Unit Activation Potentials } \\
\hline & Insertional Activity & Fibrillation Potentials & Fasciculations & Amplitude & Duration & Polyphasia & Activation & Recruitment \\
\hline R Iliopsoas & NL & None & None & $\uparrow$ & NL & $\uparrow$ & NL & NL \\
\hline R Vastus medialis & $\uparrow \uparrow$ & 2 & None & $\uparrow$ & NL & $\uparrow$ & NL & NL \\
\hline R Adductor longus & $\uparrow \uparrow \uparrow$ & 3 & None & NL & NL & NL & NL & $\downarrow \downarrow \downarrow$ \\
\hline R Tensor fasciae latae & NL & None & None & NL & NL & NL & NL & NL \\
\hline R Tibialis anterior & NL & None & None & NL & NL & NL & NL & NL \\
\hline
\end{tabular}

Key: $\uparrow$, slightly increased; $\uparrow \uparrow$, moderately increased; $\uparrow \uparrow \uparrow$, severely increased ; $\downarrow \downarrow \downarrow$, severely reduced; NL, normal

Table 3. Nerve Conduction Studies - March 2016.

\begin{tabular}{|c|c|c|c|c|c|c|c|c|c|c|c|c|c|}
\hline \multirow[t]{2}{*}{ Nerve Stimulated } & \multirow[t]{2}{*}{ Stimulation Site } & \multirow[t]{2}{*}{ Recording Site } & \multicolumn{3}{|c|}{ Amplitude* } & \multicolumn{3}{|c|}{ Latency (ms) } & \multicolumn{3}{|c|}{ Conduction Velocity (m/s) } & \multirow[t]{2}{*}{ F wave Latency (ms) } & \multirow[t]{2}{*}{ Temperature $\left({ }^{\circ} \mathrm{C}\right)$} \\
\hline & & & RT & LT & NL & RT & LT & NL & RT & LT & NL & & \\
\hline \multirow[t]{3}{*}{ Peroneal (m) } & Ankle & EDB & NR & & $\geq 2.0$ & NR & & $\leq 6.6$ & & & & Not performed & \\
\hline & Below fibula & EDB & NR & & & NR & & & NR & & $\geq 42.0$ & & \\
\hline & Lateral popliteal fossa & EDB & NR & & & NR & & & NR & & $\geq 42.0$ & & 31.2 \\
\hline \multirow[t]{2}{*}{ Peroneal (m) } & Below fibula & TA & 0.1 & & $\geq 5.0$ & 5.36 & & $\leq 6.6$ & & & & Not performed & \\
\hline & Lateral popliteal fossa & TA & 0.1 & & & 7.45 & & & 48 & & $\geq 42.0$ & & 31.2 \\
\hline \multirow[t]{2}{*}{ Tibial (m) } & Ankle & AHB & 0.2 & & $\geq 2.0$ & 8.28 & & $\leq 6.6$ & & & & Not performed & \\
\hline & Popliteal fossa & AHB & 0.2 & & & 22.9 & & & 27.3 & & $\geq 42.0$ & & 31.2 \\
\hline Sural (s) & Calf & Posterior ankle & NR & & $\geq 5.0$ & NR & & $\leq 4.2$ & NR & & $\geq 42.0$ & & 31.2 \\
\hline
\end{tabular}

* Amplitude: motor in millivolts; sensory in microvolts

Key: m, motor study; s, sensory study; RT, right; LT, left; NL, normal; EDB, extensor digitorum brevis; TA, tibialis anterior; AHB, abductor hallucis brevis; NR, no response Note: sensory latencies are peak latencies

Table 4. Electromyography - March 2016.

\begin{tabular}{|c|c|c|c|c|c|c|c|c|}
\hline \multirow[b]{2}{*}{ Muscle } & \multicolumn{3}{|c|}{ Spontaneous Activity } & \multicolumn{5}{|c|}{ Voluntary Motor Unit Activation Potentials } \\
\hline & Insertional Activity & Fibrillation Potentials & Fasciculations & Amplitude & Duration & Polyphasia & Activation & Recruitment \\
\hline R Vastus lateralis & $\uparrow \uparrow$ & 2 & None & $\uparrow$ & $\uparrow \uparrow$ & $\uparrow \uparrow$ & NL & $\downarrow \downarrow \downarrow$ \\
\hline R Rectus femoris & $\uparrow \uparrow$ & 2 & None & - & - & - & Absent & - \\
\hline R Tibialis anterior & $\uparrow \uparrow \uparrow \uparrow$ & 4 & None & $\downarrow$ & 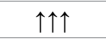 & $\uparrow \uparrow \uparrow$ & NL & $\downarrow \downarrow \downarrow$ \\
\hline R Gastrocnemius (Medial head) & $\uparrow \uparrow$ & 2 & None & NL & NL & $\uparrow$ & NL & $\downarrow$ \\
\hline R Thoracic paraspinals (mid) & NL & None & None & NL & NL & NL & NL & NL \\
\hline R Thoracic paraspinals (low) & NL & None & None & NL & NL & NL & NL & NL \\
\hline R Lumbar paraspinals (low) & $\uparrow$ & 1 & None & - & - & - & Poor & - \\
\hline L Rectus femoris & NL & NL & NL & NL & NL & NL & NL & NL \\
\hline L Tibialis anterior & $\uparrow$ & 1 & None & NL & NL & NL & NL & $\downarrow$ \\
\hline
\end{tabular}

Key: $\uparrow$, slightly increased; $\uparrow \uparrow$, moderately increased; $\uparrow \uparrow \uparrow$, severely increased ; $\downarrow$, slightly reduced; $\downarrow \downarrow \downarrow$, severely reduced; NL, normal; -, not scored 

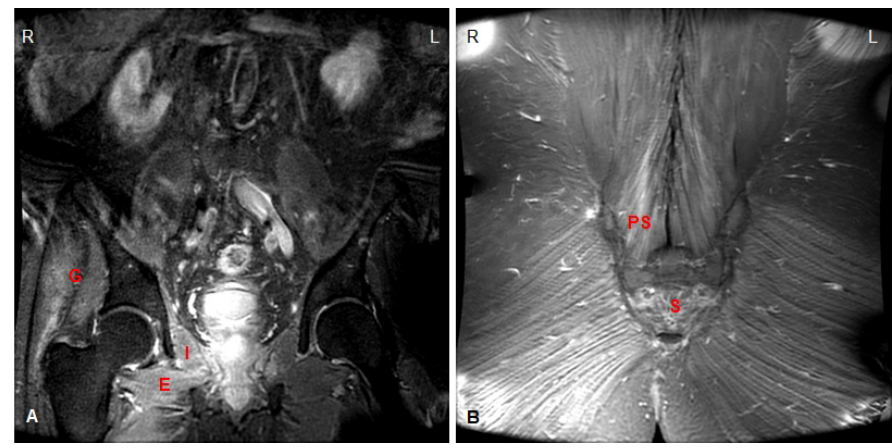

Figure 2. MRI of Pelvis (2016). (A) Coronal STIR hyperintensities demonstrated, most prominent within the right gluteus maximus/medius (G), obturator externus (E), and obturator internus (I) muscles.

(B) Coronal STIR hyperintensities demonstrated, most prominent within the right lower lumbar paraspinal muscles (PS); fatty replacement within sacrum (S).

Radiotherapy may cause an (initially) asymptomatic microvascular injury to exposed tissues, related to an endothelial cell-mediated inflammatory response to increased oxidative stress. Over time, cytokine-driven fibroblast proliferation and extracellular matrix deposition incite a cycle of fibrosis, further perpetuated by the continued production of reactive oxygen species. Late (often monthsto-years) after radiotherapy, a terminal disease phase, characterized by irreversible ischemia, fibroatrophy, and finally tissue necrosis, develops [1].

Several treatment-related factors elevating the risk of radiationinduced neuropathy are known, including a large total dose (i.e. $>50$ Gy to a nerve plexus), dose per fraction or hot spot, treatment fields encompassing a high concentration of nerve fibers, body positioning, and combined treatment with neurotoxic chemotherapeutic agents or surgery $[1,2]$. Patient-related risk factors for delayed nerve injury include, as in the case of our patient, advanced age, diabetes (with or without an associated neuropathy), other cardiovascular comorbidities, and tobacco use [1]. Treatment is currently supportive.

In patients with both early and locally advanced disease, externalbeam radiation therapy is considered a definitive therapy for prostate cancer, and held to be as effective in stage T1 and T2 disease as other widely used modalities, including radical prostatectomy, brachytherapy, and androgen deprivation therapy [3]. According to commonly cited stratification schemes used to guide treatment recommendations and predict prostate cancer recurrence, our patient was considered to be at intermediate risk $[4,5]$. He opted for definitive radiotherapy, and his cancer was successfully treated with a standard radiation dosing and fractionation schedule.

Due to the delayed clinical presentation of radiculoplexus neuropathy, often many years after radiotherapy is complete, the association with this exposure may initially be missed [1], thereby delaying diagnosis, and potentially exposing patients to unnecessary testing and ineffective treatments [1]. Our patient was initially recognized as a poorly controlled diabetic, and in this context, was treated for presumed diabetic amyotrophy - a generally monophasic microvasculitis of acute-to-subacute onset, accompanied by pain (a painless motor form exists) [6]. Reevaluation was delayed by several months after treatment, by which time his progression and corticosteroid failure were apparent. Among the pertinent differential considerations excluded in his case were structural/mass lesions of the lumbosacral spine and pelvis, and biochemical radiotherapy failure (considered unlikely given persistently low-normalized PSA values post-treatment) [7]. Given his previous radiotherapy sensitivities, in the absence of other probable culprits, the diagnosis of delayed radiation-induced radiculoplexopathy was made.

This case underscores the need for a high index of suspicion for delayed neurotoxicity in susceptible patients previously exposed to pelvic radiation presenting with progressive lower extremity deficits. Recognition of this often-under-appreciated phenomenon is critical to providing patients with realistic goals and supportive therapies, and in regards to cancer treatment planning and counseling.

\section{Acknowledgments}

Neil W. Kowall, MD, for his guidance and general support of this manuscript submission

VA Boston Healthcare Service, Chief of Neurology Service, Boston University School of Medicine, Professor of Neurology, Boston, Massachusetts, USA

\section{Competing interests}

The author(s) declare that they have no competing interests.

\section{References}

1. Delanian S, Lefaix JL, Pradat PF (2012) Radiation-induced neuropathy in cancer survivors. Radiother Oncol 105: 273-282. [Crossref]

2. Jaeckle KA (2010) Neurologic manifestations of neoplastic and radiation-induced plexopathies. SeminNeurol 30: 254-262.[Crossref]

3. Nguyen PL, Aizer A, Assimos DG, D’Amico AV, Frank SJ, et al. (2014) American College of Radiology, Expert Panel on Radiation Oncology-Prostate. ACR Appropriateness Criteria ${ }^{\circledR}$ Definitive External-Beam Irradiation in stage T1 and T2 prostate cancer. Am J Clin Oncol 37: 278-288. [Crossref]

4. D'Amico AV, Whittington R, Malkowicz SB, Schultz D, Blank K, et al. (1998) Biochemical outcome after radical prostatectomy, external beam radiation therapy, or interstitial radiation therapy for clinically localized prostate cancer. JAMA 280: 969974. [Crossref]

5. Mohler JL, Armstrong AJ, Bahnson RR, D’Amico AV, Davis BJ, et al. (2016) Prostate Cancer, Version 1.2016. J Natl Compr Canc Netw 14: 19-30. [Crossref]

6. Garces-Sanchez M, Laughlin RS, Dyck PJ, Engelstad JK, Norell JE, et al. (2011) Painless diabetic motor neuropathy: a variant of diabetic lumbosacral radiculoplexus Neuropathy? Ann Neurol 69: 1043-1054. [Crossref]

7. Roach M 3rd, Hanks G, Thames H Jr, Schellhammer P, Shipley WU, et al. (2006) Defining biochemical failure following radiotherapy with or without hormonal therapy in men with clinically localized prostate cancer: recommendations of the RTOGASTRO Phoenix Consensus Conference. Int J Radiat Oncol Biol Phys 65: 965-974. [Crossref]

Copyright: (C2017 Domingo-Horne RM. This is an open-access article distributed under the terms of the Creative Commons Attribution License, which permits unrestricted use, distribution, and reproduction in any medium, provided the original author and source are credited. 